\title{
Responses of young girls to two modes of aerobic training
}

\author{
Joanne R Welsman, Neil Armstrong, Sandra Withers
}

\begin{abstract}
Objectives-To investigate the physiological effects of two different three times a week, eight week training programmes on the aerobic fitness of nine to ten year old girls.

Methods-Treadmill determined peak
\end{abstract} $\dot{\text { Vo }}{ }_{2}$, submaximal heart rates, and submaximal blood lactate were the criterion measures. Seventeen girls completed a programme of "aerobics" training where sessions lasted 20-25 minutes. Eighteen girls followed a cycle ergometer training programme which involved pedalling continuously for 20 minutes with the heart rate maintained between 160 and 170 beats/minute. A control group of 16 girls completed the criterion tests but did not train. In the cycle ergometer group and eight control subjects plasma total cholesterol and high density lipoprotein cholesterol were determined before and after training.

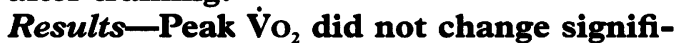
cantly with training in either training group, neither were there any significant changes in submaximal heart rates. Blood lactate declined significantly at the two lowest submaximal exercise intensities in the cycle ergometer training group (from $2.3(1.1)$ to $1.4(0.06) \mathrm{mmol} / \mathrm{h}$ at stage 1 and from $2.1(1.2)$ to $1.6(0.06) \mathrm{mmol} / \mathrm{h}$ at stage 2; means (SD); $\mathbf{P}<0.01)$. Total cholesterol and high density lipoprotein cholesterol remained unchanged with training.

Conclusions-These findings suggest that an eight week structured exercise programme produces minimal changes in either the aerobic fitness or blood lipids of young girls. It may be more beneficial for long term health to promote enjoyment in activity and positive attitudes to exercise rather than attempting to enhance aerobic fitness through strenuous exercise programmes.

(Br J Sports Med 1997;31:139-142)

Keywords: children; training; aerobic fitness; blood lipids

It is well documented that exercise training according to appropriate guidelines ${ }^{1}$ will elicit significant increases in peak oxygen uptake (peak $\dot{\mathrm{V}}_{2}$ ) and reductions in submaximal $\dot{\mathrm{V}}_{2}$ and blood lactate in untrained adults. ${ }^{2 \frac{2}{3}}$ Despite considerable research, however, the responses of young children to aerobic exercise training are not fully understood. It has been suggested that a maturational threshold ${ }^{4}$ or "trigger point" exists below which children are unable to improve aerobic fitness. Others have argued that there is no evidence that prepubescent children are any less responsive to training than older individuals. ${ }^{6} \mathrm{We}^{7}$ and others ${ }^{8}$ have reviewed the few studies that have used appropriate methodologies and training programmes and included a control group, and concluded that young children can respond to structured exercise training but the magnitude of any change in aerobic fitness is likely to be considerably less than expected in adolescents and adults.

Several studies have reported significant increases in the peak $\dot{\mathrm{VO}}_{2}$ of young boys after training programmes of at least eight weeks' duration ${ }^{10}$ but an absence of change has also been reported. ${ }^{11}$ Training four times a week for 12 weeks elicited a $11.4 \%$ increase in the peak $\dot{\mathrm{VO}}_{2}$ (litres/min) of 11 boys and five girls compared with a $7.1 \%$ increase in the control group. ${ }^{12}$ In contrast, an enhanced physical education programme which included 25 minutes of physical activity four days a week for 12 weeks failed to increase the peak $\dot{\mathrm{Vo}}_{2}$ of 11 seven to nine year old boys and girls. ${ }^{4}$ However, these findings with mixed sex groups are difficult to interpret given the significantly higher peak $\dot{\mathrm{VO}}_{2}$ of young boys compared with girls. ${ }^{4}$ No well controlled studies of the trainability of the aerobic fitness of young non-athletic girls appear to be available. ${ }^{8}$

Studies with adults have demonstrated beneficial effects of physical activity upon blood lipid and lipoprotein profiles, ${ }^{13-15}$ although contrasting results have also been reported. ${ }^{16-18}$ Many confounding variables interact with blood lipid levels - for example, age, body mass and fatness, cigarette smoking, diet, which may have contributed to the conflicting findings, but recent meta-analyse ${ }^{19} 20$ have concluded that physical activity does favourably improve blood lipid and lipoprotein profiles with the most important changes being increased high density lipoprotein (HDL) cholesterol, $\mathrm{HDL}_{2}$ cholesterol, and apolipoprotein A-1 concentrations. ${ }^{21}$ Data from studies with adolescents are sparse relative to the volume of adult literature, and the results are confounded by imprecise methods of estimating physical activity and the dynamic changes in blood lipoproteins that occur during adolescence. Despite indications of higher HDL cholesterol in active compared with inactive adolescents, prospective training studies have failed to elicit favourable changes in blood lipid or lipoprotein profiles. $^{22}$ 
The findings of the few prospective training studies with young children have been equivocal. ${ }^{7}$ Several studies have reported favourable, although sometimes transient, changes in blood lipids, ${ }^{102324}$ whereas exercise training or increased physical activity has failed to alter lipid and lipoproteins in others. ${ }^{9} 1027$ Once again, few data are available for young girls and there is a need for further investigation.

In response to the lack of data describing the physiological responses of young girls to aerobic exercise, the aim of the present study was to examine the effect of two different modes of aerobic training upon the aerobic fitness and blood lipid levels of nine to ten year old girls.

\section{Methods}

After gaining approval from the local Health Trust Ethical Committee an initial approach was made to all of the girls (approximately 100) in Year 5 of two local Middle schools. Letters describing the project and consent forms were issued to all those expressing an interest in taking part. From those who returned consent forms, signed by both parent/guardian and child, 30 children were randomly selected from each school. Within each school, 20 children were randomly assigned to the training group. The training group from one school completed cycle ergometer training while the other group trained using a variety of aerobic activities to music (aerobics). The specific training programmes are described below. The remaining 20 girls formed the control group. Five children failed to complete the training programme satisfactorily and four of the control group did not return for the second phase of laboratory tests. Results are thus presented for the remaining 51 children.

Before the start of the project all subjects spent a morning at the Research Centre for familiarisation with laboratory testing procedures and to practise treadmill running. Each child also received a brief medical examination from an experienced nurse to evaluate current health status and estimate the child's maturity as indicated by pubic hair development. ${ }^{28}$ Subsequently, children visited the Research Centre in small groups to complete a range of physiological tests, which were repeated after the training programmes.

Stature was measured to the nearest $0.01 \mathrm{~m}$ using an Holtain stadiometer (Crymych, Dyfed, Wales, UK), and body mass was measured to the nearest $0.1 \mathrm{~kg}$ using an Avery balance beam (Avery, Birmingham, UK). Peak oxygen uptake (peak $\dot{\mathrm{VO}}_{2}$ ) was measured during a progressive treadmill running test to exhaustion. After a three minute warm up at 6 $\mathrm{km} / \mathrm{h}$, the test protocol started at a belt speed of $7 \mathrm{~km} / \mathrm{h}$. Three-minute exercise bouts were separated by rest periods of one minute. Belt speed was increased by $1 \mathrm{~km} / \mathrm{h}$ increments to 9 $\mathrm{km} / \mathrm{h}$ for the first two stages. Thereafter, belt speed remained constant and at each stage the gradient was raised by $2.5 \%$ until the subject was unable to continue. Strong verbal encouragement was given throughout the test. Peak
$\dot{\mathrm{V}}_{2}$ was accepted as a maximal index if the child showed clear signs of intense effort (unsteady gait, sweating, hyperpnoea, facial flushing, grimacing), attained a heart rate within $5 \%$ of age predicted maximum or showed a levelling in heart rate over the final stages, and respiratory exchange ratio (RER) was at least $1.0 .^{729}$ Ventilation and expired oxygen and carbon dioxide were continuously monitored via a computerised on line gas analysis system (Oxycon Sigma, Cranlea Ltd, Birmingham, UK). The system was calibrated according to the manufacturer's recommendations before each test using gases of known concentration. Heart rate was monitored throughout the test using an electrocardiograph (Rigel, Morden, UK). Blood was sampled from a warmed fingertip after each exercise stage and immediately after peak $\dot{\mathrm{V}}_{2}$ for immediate whole blood lactate determination (YSI 2300 stat plus; Yellow Springs Instruments, Yellow Springs, OH, USA). A fingertip blood sample was also obtained before exercise from the children in the cycle training group and the control subjects from the same school. The sample was centrifuged for five minutes and the plasma drawn off and stored on ice. Plasma total cholesterol and HDL cholesterol were determined using the cholesterol oxidase method (Kodak Ektachem 700, Rochester, NY, USA).

\section{TRAINING PROGRAMMES \\ Cycle ergometer training}

Children trained on Monark cycle ergometers three times per week for a period of eight weeks. After a five minute warm up of unloaded pedalling, the training period lasted 20 minutes at an individually determined exercise intensity which maintained heart rate between 160 and 170 beats $/ \mathrm{min}$. In children of this age, this approximates to $80 \%$ peak heart rate and is in accordance with current guidelines for increasing aerobic fitness. ${ }^{7}$ Each child's heart rate was monitored continuously throughout every training session using cardiometers and all sessions were supervised by a member of the research team (SW).

\section{Aerobics group}

Training sessions were held three times per week at the school by a member of the research team (JRW). Each session lasted approximately 40 minutes. Twice a week the body of the session (20 to 25 minutes) consisted of a variety of floor and step aerobic routines to music and aerobic games. The third session was based around the use of circuit equipment with a variety of exercises which included aerobic, strength, and muscular endurance components. All sessions started with a five to ten minute aerobic warm up during which intensity was gradually increased. Sessions ended with a similar warm down period and some flexibility work.

Before the training study, pilot work was carried out with 27 girls aged nine to ten years to evaluate the heart rate response to both the aerobic routines and isolated movements within the routines. This demonstrated that the 
Table 1 Descriptive statistics of girls by group. Values are mean (SD)

\begin{tabular}{llll}
\hline & Aerobics $(n=17)$ & Cycle ergometer $(n=18)$ & Control $(n=16)$ \\
\hline $\begin{array}{llll}\text { Age (years) } \\
\quad \text { Pre }\end{array}$ & $10.2(0.3)$ & $10.1(0.5)$ & $10.2(0.4)$ \\
$\quad$ Post & $10.3(0.3)$ & $10.3(0.5)$ & $10.3(0.4)$ \\
Stature (m) & & $1.41(0.06)$ & $1.40(0.09)$ \\
$\quad$ Pre & $1.37(0.07)$ & $1.43(0.06)$ & $1.41(0.09)$ \\
$\quad$ Post & $1.39(0.07)$ & $34.0(3.7)$ & $37.2(11.1)$ \\
Mass (kg) & $33.6(6.9)$ & $34.3(3.9)$ & $37.5(11.1)$ \\
$\quad$ Pre & $33.7(6.7)$ & & \\
Post & & & \\
\hline
\end{tabular}

Table 2 Peak exercise responses and blood lipid pre-and post-training. Values are mean $(S D)$

\begin{tabular}{|c|c|c|c|}
\hline & Aerobics $(n=17)$ & Cycle ergometer $(n=18)$ & Control $(n=16)$ \\
\hline \multicolumn{4}{|c|}{ Peak $\dot{V O}_{2}$ (litres/min) } \\
\hline Pre & $1.58(0.26)$ & $1.76(0.18)$ & $1.72(0.35)$ \\
\hline Post & $1.61(0.23)$ & $1.79(0.14)$ & $1.72(0.37)$ \\
\hline \multicolumn{4}{|c|}{ Peak heart rate (beats/min) } \\
\hline Pre & $208(8)$ & $210(6)$ & $208(10)$ \\
\hline Post & $206(7)$ & $203(4.3)^{\star}$ & $209(8)$ \\
\hline \multicolumn{4}{|c|}{ Peak RER } \\
\hline Pre & $1.05(0.04)$ & $1.1(0.04)$ & $1.08(0.06)$ \\
\hline Post & $1.05(0.04)$ & $1.05(0.03)$ & $1.07(0.04)$ \\
\hline \multicolumn{4}{|c|}{ Peak blood lactate $(\mathrm{mmol} / \mathrm{l})$} \\
\hline Pre & $6.5(1.4)$ & $7.6(1.9)$ & $7.6(2.4)$ \\
\hline Post & $6.6(1.2)$ & $6.1(1.8)$ & $7.4(1.7)$ \\
\hline \multicolumn{4}{|c|}{ Total cholesterol $(\mathrm{mmol} / \mathrm{l})$} \\
\hline $\begin{array}{l}\text { Pre } \\
\text { Post }\end{array}$ & Not sampled & $\begin{array}{l}5.12(0.75) \\
4.67(0.58)\end{array}$ & $\begin{array}{l}5.50(1.05) \dagger \\
4.96(1.05)\end{array}$ \\
\hline \multicolumn{4}{|c|}{ HDL cholesterol $(\mathrm{mmol} / \mathrm{l})$} \\
\hline Pre & Not sampled & $1.70(0.88)$ & $1.67(0.42) \dagger$ \\
\hline Post & & $1.50(0.43)$ & $1.69(0.27)$ \\
\hline
\end{tabular}

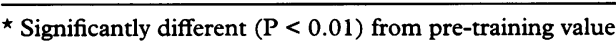

$+\mathrm{n}=8$.

RER, respiratory exchange ratio.
There were no significant differences $(\mathrm{P}>0.05)$ in age, stature, mass, or peak $\mathrm{V}_{2}$ between the three subject groups before training.

All children in the training groups completed the 24 exercise sessions. Table 2 gives the preand post-training peak exercise responses. Peak $\dot{\mathrm{V}}_{2}$ and peak RER did not change significantly $(P>0.05)$ in any group during training. The peak heart rate of the cycle trained group declined significantly $(P<0.01)$. No significant $(P>0.05)$ changes in heart rate during the first five submaximal stages of the treadmill test were noted in any group. The blood lactate measured during the first two submaximal stages of the treadmill test decreased significantly $(P<0.01)$ in the cycle trained group (from $2.3(1.1)$ to $1.4(0.06) \mathrm{mmol} / \mathrm{l}$ at stage 1 and from $2.1(1.2)$ to $1.6(0.06) \mathrm{mmol} / \mathrm{l}$ at stage 2 ; means (SD)) but no other significant differences were noted. In the cycle ergometer training group, there were no significant $(P>0.05)$ changes in either plasma total cholesterol or HDL cholesterol.

\section{Discussion}

Few studies have investigated the responses of young children to structured exercise, and there is a notable lack of data for young girls. ${ }^{78}$ One reason for this might be the problem of retaining young children's interest in an exercise programme for an extended period of time, and previous studies have suffered attrition rates as high as $63 \% .{ }^{4}$ The attrition rate of $12.5 \%$ in the training group in the present study is remarkably low and explained by genuine reasons such as illness or absence from school rather than disinterest in the training.

The key finding of the present study was the absence of change in peak aerobic fitness in either training group after the eight week training programme. Well controlled training studies including young girls against which to compare these findings are sparse. ${ }^{7822}$ Two previous studies which have included prepubertal girls have produced conflicting conclusions. Lussier and Buskirk ${ }^{12}$ reported a significant increase in the absolute peak $\dot{\mathrm{V}}_{2}$ of a group of 11 children, which included five girls, after 12 weeks of running training. This was despite pretraining levels of peak $\dot{\mathrm{VO}}_{2}$ similar to those in the present study. However, a second study, ${ }^{4}$ which included six boys and five girls, failed to find any significant increase in peak $\dot{\mathrm{V}} \mathrm{O}_{2}$ after a 12 week period of increased physical activity.

In adults, the magnitude of the increase in peak $\mathrm{V}_{2}$ with training is influenced by the pretraining levels of aerobic fitness. Where these are already high, training-induced improvements in aerobic function may only be detectable in submaximal responses. ${ }^{30}$ In the present study, neither mode of training elicited any favourable changes in submaximal heart rate responses. With continuous cycle training there was a trend towards a reduction in submaximal blood lactate levels, but only at the first two treadmill stages $(7$ and $8 \mathrm{~km} / \mathrm{h})$ did these achieve significance. Greater submaximal changes might have been apparent had the were classified as Tanner stage one for pubic hair. One child in each training group was stage 2. Twelve of the control children were Tanner stage 1 , three were stage 2 and one was stage 4 . 
training mode more closely reflected the testing mode (treadmill running); however, these findings do indicate that improvements in aerobic function in young children may be more likely at submaximal rather than maximal exercise intensity.

The levels of total cholesterol and HDL cholesterol measured in the present subjects are in accordance with previous reports. ${ }^{31}$ Only one other study appears to have examined blood lipid changes in young girls. ${ }^{24}$ Gilliam and Burke ${ }^{24}$ found no change in total cholesterol, but a significant increase in HDL cholesterol after six weeks of five $\mathbf{4 0}$ minute sessions of physical activity. The absence of change in total cholesterol after training in the cycle trained girls in this study is in agreement with this, but no changes in HDL were identified despite the two week longer duration of the exercise programme. Other studies including smaller mixed sex groups have also failed to change blood lipid profiles with $\operatorname{ten}^{4}$ and twelve $^{25}$ week training programmes. On balance, it would appear that a structured exercise programme provides minimal benefits to the total and HDL cholesterol levels of young girls with normal blood lipid levels.

In conclusion, the present study suggests that vigorous training will make minimal changes to peak or submaximal aerobic function in young girls. Neither does exercise training appear to benefit blood lipid levels. Given these findings and the evidence that it is children's low levels of habitual physical activity that are more cause for concern than their aerobic fitness, ${ }^{32} 33$ it may be more important for children's future health and well being to focus upon encouraging them to adopt more active lifestyles and develop positive attitudes towards physical activity rather than emphasising exercise training to enhance current levels of aerobic fitness.

1 American College of Sports Medicine. Position stand on the recommended quantity and quality of exercise for developing and maintaining cardiorespiratory and muscular fitness in healthy adults. Med Sci Sports Exerc 1990;22:265-74.

2 Gaesser GA, Poole DC. Lactate and ventilatory thresholds: disparity in time course of adaptations to training. $\mathcal{F} A p p l$ Physiol 1986;61:999-1004.

3 Poole DC, Gaesser GA. Response of ventilatory and lactate thresolds to continuous and interval training. $f$ Appl Physiol 1985;58:1115-21.

4 Gilliam TB, Freedson PS. Effects of a 12 week school physical fitness program on peak $\mathrm{Vo}_{2}$, body composition and cal fitness program on peak $\mathrm{VO}_{2}$, body composition and blood lipids in

5 Katch VL. Physical conditioning of children. $\mathcal{f}$ Adoles Health Care 1983;3:241-6.

6 Shephard RJ. Effectiveness of training programmes for prepubescent children. Sports Med 1992;13:194-213.

7 Armstrong N, Welsman J. Young people and physical activity. Oxford: Oxford University Press, 1997:122-36.

8 Pate RR, Ward DS. Endurance exercise trainability in children and youth. In: Grana WA, Lombardo JA, Sharkey
BJ, Stone JA, editors. Advances in sports medicine and fitness, Vol 3. Chicago: Year Book Publishers, 1990:37-55.

9 Rotstein A, Dotan R Bar-Or O, Tenebaum G. Effect of Rotstein A, Dotan R, Bar-Or O, Tenebaum G. Effect of
training on anaerobic threshold, maximal aerobic power and anaerobic performance of preadolescent boys. Int $f$ Sports Med 1986;7:281-6.

10 Savage MP, Petratis MM, Thomson WH, Berg K, Smith JL, Sady SP. Exercise training effects on serum lipids of prepubescent boys and adult men. Med Sci Sports Exerc 1986;18: 197-204.

11 Becker DM, Vaccaro P. Anaerobic threshold alterations caused by endurance training in young children. $f$ Sports Med Phys Fitness 1983;23:445-9.

12 Lussier L, Buskirk ER. Effects of an endurance training regimen on assessment of work capacity in prepubertal regimen on assessment of work capacity in
children. Ann N Y Acad Sci 1977;30:734-47.

13 Williams PT, Krauss RM, Vranizan KM, Wood PD Changes in lipoprotein subfractions during diet-induced and exercise-induced weight loss in moderately overweigh men. Circulation 1990;81:1293-304

14 Wood PD, Stefanick ML, Dreon DM, Fray-Hewitt B, Garay SC, Williams PT, et al. Changes in plasma lipids and lipoproteins in overweight men during weight loss through dieting as compared with exercise. N Engl f Med 1988;319: 1173-9.

15 Wood PD, Stefanick ML, Williams PT, Haskell WL. The effects on plasma lipoproteins of a prudent weighteffects on plasma lipoproteins of a prudent weightand women. $N$ Engl $\mathcal{f}$ Med 1991;325:461-6.

16 Gaesser GA, Rich RG. Effects of high and low intensity exercise training on aerobic capacity and blood lipids. Med Sci Sports Exerc 1984;16:269-74.

17 Hartung GH, Foreyt JP, Mitchell RE, Vlasek I, Gotto AM Relation of diet to high intensity lipoprotein cholesterol in middle aged marathon runners, joggers and inactive men. N Engl f Med 1980;302:357-61.

18 Peltonen P, Marnierni J, Hietanen E, Vuori I, Ehnholm C. Changes in serum lipids, lipoproteins and heparin releasable lipolytic enzymes during moderate physical training in man: a longitudinal study. Metabolism 1981;30:518-20.

19 Lokey EA, Tran ZV. Effects of exercise training on serum Lokey EA, Tran ZV. Effects of exercise training on serum
lipid and lipoprotein concentrations in women: a metalipid and lipoprotein concentrations in
analysis. Int $₹$ Sports Med 1989;10:424-9.

20 Tran ZV, Weltman A, Glass GV, Mood DP. The effects of exercise on blood lipids and lipoproteins a meta-analysis of studies. Med Sci Sports Exerc 1983;15:393-402.

21 Durstine JL, Haskell WL. Effects of exercise training on plasma lipids and lipoproteins. Exerc Sport Sci Rev 1994;22:477-521.

22 Armstrong N, Simons-Morton B. Physical activity and blood lipids in adolescents. Pediatric Exercise Science 1994;6:381-405.

23 Fisher AG, Brown $M$. The effects of diet and exercise on selected coronary risk factors in children. Med Sci Sport Exerc 1982;14:2

24 Gilliam TB, Burke MB. Effect of exercise on serum lipids and lipoproteins in girls, ages 8 to 10 years. Artery 1978;4: 203-13.

25 Ben-Ezra V, Gallagher K. Blood lipid profiles of 8-10 year old children: the effect of diet and exercise. Med Sci Sports Exerc 1989;21:584.

26 Dwyer T, Coonan WE, Leitch DR, Hetzel BS, Baghurst RA. An investigation of the effects of daily physical activity on the health of primary school students in South Australia. Int $f$ Epidemiol 1983;12:308-13.

27 Armstrong N, Kirby BJ, McManus AM, Welsman JR. Aerobic fitness of prepubescent children. Ann Hum Biol 1995;22:427-41.

28 Tanner JM. Growth at adolescence. 2nd ed. Oxford: Blackwell Scientific Publications, 1962.

29 Armstrong N, Welsman J. Assessment and interpretation of aerobic fitness in children and adolescents. Exerc Sport Sci $\operatorname{Rev} 1994 ; 22: 435-76$.

30 Sjödin B, Jacobs I, Svedenhag J. Changes in onset of blood lactate accumulation and muscle enzymes after training OBLA. Eur ₹ Appl Physiol 1982;49:45-57.

31 Armstrong N, Balding J, Gentle P, Kirby B. Serum lipids and blood pressure in relation to age and sexual maturity. and Hum Biol 1992;19:477-87.

32 Armstrong N, Balding J, Gentle P, Williams J, Kirby B. Peak oxygen uptake and habitual physical activity in 11 to 16 oxygen uptake and habitual physical activity in 11

33 Armstrong N, McManus A, Welsman J, Kirby B. Physical activity patterns and aerobic fitness among pre-pubescents. European Physical Education Reviews 1996;2:19-29. 